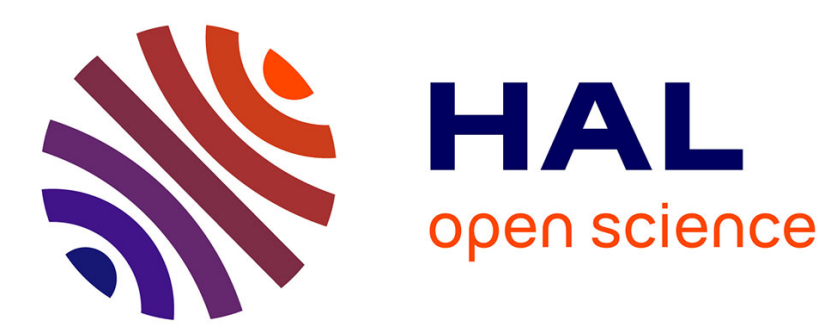

\title{
Unconventional GMR angular dependence using a compensated ferrimagnet
}

\author{
N.T. Nam, Laurent Ranno
}

\section{To cite this version:}

N.T. Nam, Laurent Ranno. Unconventional GMR angular dependence using a compensated ferrimagnet. Journal of Magnetism and Magnetic Materials, 2010, 322 (9-12), pp.1428-1430. 10.1016/j.jmmm.2009.07.007 . hal-00635164

\section{HAL Id: hal-00635164 \\ https://hal.science/hal-00635164}

Submitted on 28 Oct 2011

HAL is a multi-disciplinary open access archive for the deposit and dissemination of scientific research documents, whether they are published or not. The documents may come from teaching and research institutions in France or abroad, or from public or private research centers.
L'archive ouverte pluridisciplinaire HAL, est destinée au dépôt et à la diffusion de documents scientifiques de niveau recherche, publiés ou non, émanant des établissements d'enseignement et de recherche français ou étrangers, des laboratoires publics ou privés. 
Unconventional GMR angular dependence using a compensated ferrimagnet

\title{
N. T. Nam $1,2,{ }^{*}$, L.Ranno $1,+$
}

1 Institut Néel, CNRS-UJF, Grenoble, France

2 Laboratory for Nanomagnetic Materials and Devices, Faculty of Physics, Enginering and Nanotechnology, College of Technology, Vietnam National University, Hanoi, Vietnam

* now at Dept. Eng. Phys., Le Quy Don Technical University, Hanoi, Vietnam

+ corresponding author : laurent.ranno@grenoble.cnrs.fr

\begin{abstract}
We have designed a $\mathrm{GdCo} / \mathrm{Cu} / \mathrm{NiFe}$ Giant Magnetoresistance (GMR) trilayer, the magnetoresistance of which does not always depend on the angle between the magnetisations of the electrodes. Using a GdCo ferrimagnetic alloy close to compensation, it was possible to experimentally reach the spin flop field $\mathrm{B}_{s f}$ of the ferrimagnetic layer. Below $\mathrm{B}_{s f}$, the ferrimagnetic layer behaves as a ferromagnetic layer, however above $\mathrm{B}_{s f}$, the ferrimagnetic sublattice magnetisations are no longer antiparallel and rotate $90^{\circ}$ away from the GdCo layer total magnetisation, i.e. $90^{\circ}$ away from the applied field. The GMR responds to the angle between $\mathrm{Co}$ and $\mathrm{NiFe}$ magnetisations and not to the angle between GdCo and NiFe magnetisations. Such a structure allows to study the change of sign of the GdCo spin polarisation as a function of temperature, and details of the GdCo magnetisation when usual magnetometry is difficult. FeNi can be seen as a sensor for the in-plane component of Co sublattice magnetisation, whereas extraordinary Hall effect measurements give a complementary image of the perpendicular component of the Co magnetisation.
\end{abstract}

71.20.Eh 73.50.Fq 75.47.De 75.50.Gg ferrimagnetism giant magnetoresistance compensation temperature

\section{Introduction}

The giant magnetoresistance (GMR) effect has been the subject of many fundamental and applied studies since its discovery in 1988 [1]. GMR has already had a huge industrial impact in the field of magnetic field sensing especially for data recording devices. More recently, a new sub-field of spin electronics has emerged, current induced magnetic reversal and $\mathrm{GHz}$ generation [2]. For such spin torque devices, compensated ferrimagnetic alloys such as GdCo have already been shown to be interesting model systems [9]. Their spin polarisation 
is large enough to get magnetoresistive effects [3] and they can be tuned by changing the temperature [8]. Their magnetic anisotropy can also be designed to induce out-of-plane or in-plane easy-axis depending on deposition conditions and heat treatments.

We used such a compensated ferrimagnet to get a more detailed understanding of the microscopic contributions to GMR when a ferrimagnetic electrode is used. At the same time, GMR allowed us to get a better understanding of the magnetic configuration of such a ferrimagnetic alloy in high fields close to compensation.

A ferrimagnetic material contains two magnetic sublattices antiferromagnetically coupled. It is difficult to get a direct measurement of the contributions of both sublattices to magnetisation and transport. A physical effect sensitive to only one sublattice has to be used (for example X-ray Circular Magnetic Dichroism XMCD [5], extraordinary Hall effect EHE or magneto-optical effect). However close to compensation, i.e. to the composition $\mathrm{x}_{\text {comp }}$ or temperature $\mathrm{T}_{\text {comp }}$ where both ferrimagnetic sublattice magnetisations compensate, a large magnetic field (larger than the spin flop field $\mathrm{B}_{s f}$ ) is sufficient to create a non colinear magnetic configuration in the ferrimagnet. The measurement of GMR in this regime gives new insights on the GMR effect and on the ferrimagnetic configuration.

Yang et al. [6] synthesised $\mathrm{GdCo} / \mathrm{Cu} / \mathrm{Fe}$ based GMR trilayers. They observed a GMR ratio which varies in amplitude but more surprisingly in sign with the GdCo layer thickness, keeping its nominal composition constant. They analysed this in terms of interface/volume spin-dependent scatterings.

Bai et al. [7] synthesised TMR junctions based on $\mathrm{Gd}(\mathrm{FeCo})-\mathrm{Al} 2 \mathrm{O} 3-\mathrm{FeCo}$ trilayer and also observed a change of the TMR sign at room temperature as a function of the ferrimagnetic layer composition.

In our experience, different GdCo thin layers may have similar nominal compositions but different magnetic composition due to intermixing, preferential resputtering and incorporation of impurities (oxygen). A 1\% change in the Gd content changes the compensation temperature by as much as $75 \mathrm{~K}$. We chose not to change the GdCo composition but to vary the temperature across $\mathrm{T}_{\text {comp }}$ to study the Gd-dominated and Co-dominated magnetisation regimes using the same sample.

\section{$2 \quad$ Experimental details}

$\mathrm{Fe}_{20} \mathrm{Ni}_{80}$ (bottom) $/ \mathrm{Cu} / \mathrm{Gd}_{20} \mathrm{Co}_{80}$ (top) multilayers were deposited using the dc magnetron sputtering technique. The Si substrate was kept at room temperature during deposition. Ta buffer and capping layers were added to protect the structure against oxidation. Both Ta layers act as short circuits, which would reduce the amplitude of the spin-dependent transport effects. A magnetic field was applied during deposition to induce an easy axis in the FeNi layer. Data from a $\mathrm{Si} / \mathrm{Ta}(14 \mathrm{~nm}) / \mathrm{FeNi}(10 \mathrm{~nm}) / \mathrm{Cu}(2 \mathrm{~nm}) / \mathrm{GdCo}(15 \mathrm{~nm}) / \mathrm{Ta}(8 \mathrm{~nm})$ multilayer are presented here. 


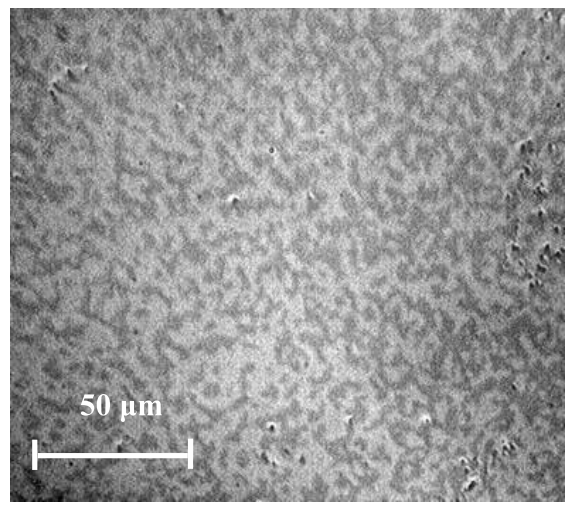

Figure 1: Zero field polar Kerr image of GdCo domains evidencing the spontaneous perpendicular magnetisation of GdCo at $300 \mathrm{~K}$

Transport measurements with the magnetic field parallel or perpendicular to the plane of the sample were performed in the range 2-300 $\mathrm{K}$ and up to 6 Tesla. Four-probe electrical configurations were used to determine the resistance $\mathrm{R}_{\square}$ and the Hall resistance $\mathrm{R}_{H}$ of the samples. Magnetisation measurements (SQUID and VSM) and magneto-optical imaging (polar Kerr microscopy at 300 $\mathrm{K})$ were also carried out to clarify the transport measurements.

\section{Magnetic configuration}

When the $\mathrm{Cu}$ spacing layer is $2 \mathrm{~nm}$-thick, both magnetic electrodes are magnetically decoupled. The $\mathrm{Fe}_{20} \mathrm{Ni}_{80}$ electrode has in-plane anisotropy with a $0.5 \mathrm{mT}$ coercivity along its field-induced easy-axis. Its out-of-plane saturation field is 1.1 Tesla, which corresponds to its demagnetising field. The GdCo layer has a spontaneous perpendicular anisotropy at room temperature due to a depositioninduced anisotropy. Polar Kerr microscopy is only sensitive to the out-of-plane component of magnetisation. The capping layer is thin enough to observe the GdCo magnetic domain structure and the image reveals a 5 micron domain structure at $300 \mathrm{~K}$ and zero-field in the demagnetised state (Fig.1).

A Hall effect loop measured at $300 \mathrm{~K}$ with the applied field perpendicular to the film (Fig. 2) evidences the spontaneous perpendicular magnetisation of GdCo and the 1.1 Tesla saturation field for FeNi. GdCo extraordinary Hall effect dominates the Hall loop. Ordinary Hall effect dominates the high-field region.

\section{Low field magnetoresistance}

When measuring the resistance of the multilayer as a function of a magnetic field applied in-plane, a large change of resistance occurs in a field larger than 


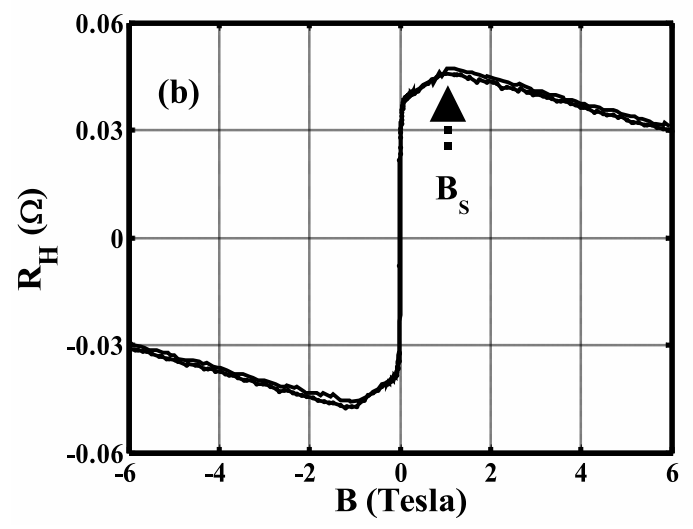

Figure 2: $300 \mathrm{~K}$ Hall effect loop measured with perpendicular applied magnetic field.

the FeNi saturation field (Fig. 3). This magnetoresistance reaches $-3 \%$ at low temperature. The saturation field reaches a maximum around $210 \mathrm{~K}$ and the magnetoresistance changes its sign (Fig. 4). $210 \mathrm{~K}$ is also the temperature where the Hall effect loop changes its sign . It corresponds to the compensation temperature of the ferrimagnetic layer [10]. The GdCo saturation field diverges because of the decrease of magnetisation and thus Zeeman energy close to $\mathrm{T}_{\text {comp }}$.

At low temperature, an inverse giant magnetoresistance effect is observed as measured by Bellouard et al. using a Gd-rich GdFeCo electrode [3]. In this temperature range the cobalt magnetisation is antiparallel to the GdCo magnetisation. Since we chose a composition which allows for compensation, at high temperature (above $\mathrm{T}_{\text {comp }}$ ), normal giant magnetoresistance is observed. One may conclude that GdCo has the same polarisation as FeNi (i.e. positive) at high temperature due to either positive spin polarisation from Co or negative spin polarisation from Gd. Below compensation, the spin polarisation reverses because both sublattices reverse compared to the net magnetisation of GdCo [8].

\section{$5 \quad$ High field magnetoresistance}

Fig. 5 shows the high field magnetoresistance below the compensation temperature for $25 \mathrm{~K}, 100 \mathrm{~K}$ and $150 \mathrm{~K}$. The resistance decreases above a critical field. The critical field decreases when heating up to $T_{\text {comp }}$ and increases again above $\mathrm{T}_{\text {comp }}$. Below $\mathrm{T}_{\text {comp }}$ the resistance vs field is not monotonous in the first quadrant above the saturation field of FeNi (1 mT).

Close to the compensation temperature, in a ferrimagnet without anisotropy, the applied magnetic field can break the antiparallel alignment above the spin flop field and $B_{s f}=\lambda\left|M_{C o}-M_{G d}\right| . \lambda$ is the molecular field coefficient which couples the $\mathrm{Gd}$ and Co sublattices. At the compensation temperature, $\mathrm{B}_{s f}$ 


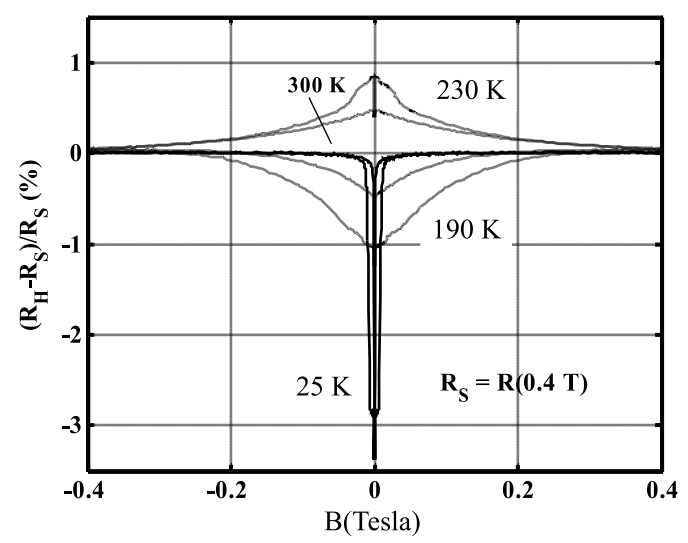

Figure 3: Magnetoresistance as a function of temperature with applied field in plane

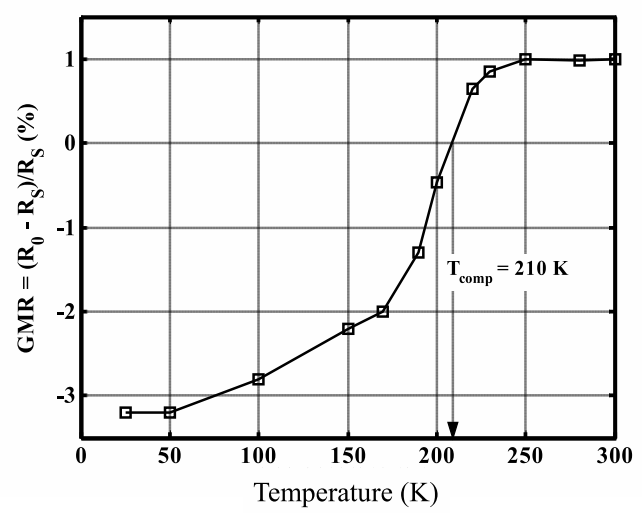

Figure 4: Amplitude of GMR as a function of temperature. The GMR is normalised to the resistance at 0.4 Tesla 


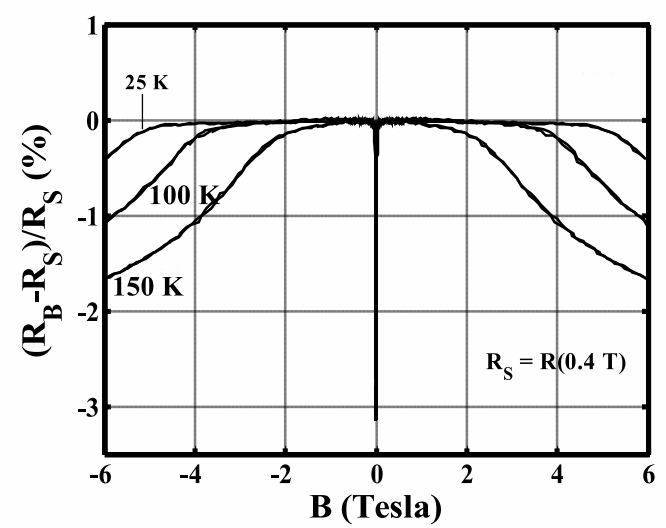

Figure 5: High field magnetoresistance evidencing the spin flop field. The field is applied in-plane. Temperatures are below the compensation temperature

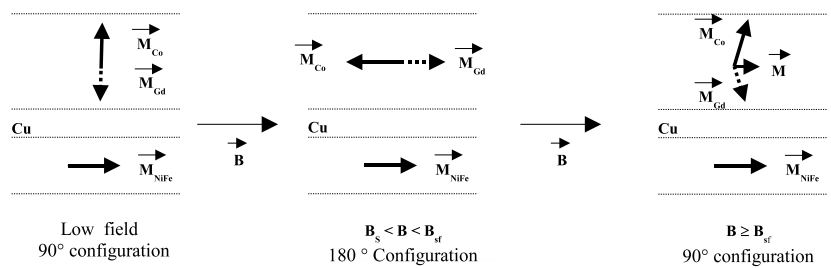

Figure 6: schematic evolution of the trilayer magnetic configuration with increasing field

goes to zero linearly with $\left|\mathrm{T}-\mathrm{T}_{\text {com }}\right|$. In our system, there is some perpendicular anisotropy so $\mathrm{B}_{s f}$ 's minimum is of the order of 2 Tesla. This value is less than the experimentally available fields.

\section{Discussion}

The GMR effect is related to the spin dependent scattering in a trilayer system. When a current passes in the plane of the multilayer (current-in-plane (cip) geometry), spin dependent scattering takes place at the interfaces and in the bulk of the magnetic electrodes. The angle dependence of the GMR effect, defining the parallel $R_{0}$ 。 and antiparallel $R_{180^{\circ}}$ resistances is then given by a cosine law.

$$
R=R_{0^{\circ}}+\frac{R_{0^{\circ}}-R_{180^{\circ}}}{2}\left(\cos \left(M_{1}, M_{2}\right)-1\right)
$$

The amplitude of the effect is given by the spin polarisations of the electrodes and decays with the thickness of the spacer [4]. Both electrodes do not always have the same sign of spin polarisations and normal GMR (same polarisation 
signs, low resistance when saturated) and inverse GMR are known to exist. High field negative magnetoresistance can be measured in systems where high magnetic fields reduce the magnetic disorder (colossal magnetoresistance system). Observing the spin flop field is a more direct proof that the antiparallel alignment is destroyed and that the measured MR is related to the magnetisation reorientation above $\mathrm{B}_{s f}$. The GdCo conduction band is mainly of Co-3d origin [11]. Gd-4f levels are far from it and do not participate in transport. The GdCo conduction band is similar to that of pure cobalt, which explains the dominant role of the cobalt sublattice.

\section{Conclusions}

Using a compensated GdCo ferrimagnetic electrode, we have synthesised a GMR trilayer. $-3 \%$ GMR is measured at low temperature. GMR changes its sign at the compensation temperature. High field measurements revealed an unusual angle dependence of the effect. Below and above the spin flop field, the GMR depends on the angle between the cobalt sublattice magnetisation and FeNi and not anymore on the angles between the magnetisations of both electrodes. These measurements show also that in this GdCo alloy cobalt dominates the GMR mechanism.

\section{Acknowledgments}

This work was supported by ACI 'ECRIN' project from CNRS. N.T.N. acknowledges a grant from the Vietnamese government (project 322).

\section{References}

[1] M. N. Baibich, J. M. Broto, A. Fert, F. Nguyen Van Dau, F. Petroff, P. Etienne, G. Creuzet, A. Friederich, and J. Chazelas Phys. Rev. Lett. 61, 2472 (1988)

[2] J. A. Katine, F. J. Albert, R. A. Buhrman, E. B. Myers, and D. C. Ralph Phys. Rev. Lett. 84, 3149 (2000)

[3] C. Bellouard, H. D. Rapp, B. George, S. Mangin, and G. Marchal, J. C. Ousset, Phys. Rev. B 53(9), p 5082-5085 (1996)

[4] T. Valet and A. Fert, Phys. Rev. B 48, 7099 (1993)

[5] L. M. Garcia, S. Pizzini, J. P. Rueff, J. Vogel, R. M. Galera, A. Fontaine, J. P. Kappler, G. Krill, and J. Goedkoop J. Appl. Phys. 79, 6497 (1996)

[6] D. Z. Yang, B. You, X. X. Zhang, T. R. Gao, S. M. Zhou, and J. Du Phys. Rev. B 74, 024411 (2006) 
[7] X. J. Bai, J. Du,J. Zhang, B. You, L. Sun, W. Zhang, X. S. Wu, S. L. Tang, and A. Hu, H. N. Hu and S. M. Zhou, J. Appl. Phys. 103, 07F305 (2008)

[8] C. Kaiser, A. F. Panchula, and S. S. P. Parkin, Phys. Rev. Lett. 95, 047202 (2005)

[9] X. Jiang,L. Gao, J. Z. Sun and S. S. P. Parkin, Phys. Rev. Lett. 97, 217202 (2006)

[10] D.H. Shin, L. Ranno, G. Suran, J. Magn. Magn. Mat. 242-245 (2002) 11781181

[11] H. Tanaka and S. Takayama, J. Appl. Phys. 70(10),p6577-6579 (1991) 